Revue internationale P.M.E.

Économie et gestion de la petite et moyenne entreprise

Revue

internationale

PME

\title{
L'assurance qualité : facteur dénaturant de la PME
}

\section{Karim Messeghem}

Volume 12, numéro 3, 1999

URI : https://id.erudit.org/iderudit/1008661ar

DOI : https://doi.org/10.7202/1008661ar

Aller au sommaire du numéro

Éditeur(s)

Presses de l’Université du Québec

ISSN

0776-5436 (imprimé)

1918-9699 (numérique)

Découvrir la revue

Citer cette note

Messeghem, K. (1999). L'assurance qualité : facteur dénaturant de la PME. Revue internationale P.M.E., 12(3), 107-126. https://doi.org/10.7202/1008661ar

\section{Résumé de l'article}

Le développement des normes ISO 9000 dans le monde touche tous les secteurs d'activité. Les dirigeants de petite entreprise peuvent s'interroger sur la pertinence de tels modèles conçus à l'origine dans des relations de type donneur d'ordres- sous-traitant. Cette contribution vise à apprécier l'impact de l'adoption d'un modèle d'assurance de la qualité sur le système de gestion de la petite entreprise. Nous montrerons dans quelle mesure la mise sous assurance qualité peut conduire à une dénaturation de la petite entreprise, autrement dit, à une remise en cause de ses principales spécificités. 


\title{
L'assurance qualité: facteur dénaturant de la PME
}

Karim MESSEGHEM

Université de Besançon

\section{MOTS CLÉS}

\section{Spécificités de la PME - Dénaturation - Assurance qualité Profil du dirigeant}

\begin{abstract}
RÉSUMÉ
Le développement des normes ISO 9000 dans le monde touche tous les secteurs d'activité. Les dirigeants de petite entreprise peuvent s'interroger sur la pertinence de tels modèles conçus à l'origine dans des relations de type donneur d'ordressous-traitant. Cette contribution vise à apprécier l'impact de l'adoption d'un modèle d'assurance de la qualité sur le système de gestion de la petite entreprise. Nous montrerons dans quelle mesure la mise sous assurance qualité peut conduire à une dénaturation de la petite entreprise, autrement dit, à une remise en cause de ses principales spécificités.
\end{abstract}

\begin{abstract}
ISO 9000 development in the world concerns all sectors. Entrepreneurs can ask themselves about the relevance of such models originally designed in principal/ subcontractor relations. The purpose of this paper is to appraise the effects of quality assurance on small firms' management systems. We show to what extent quality assurance can lead to a denaturation of small firms, in other words, a requestionning of its core specificities.
\end{abstract}

\section{L'AUTEUR}

KARIM MESSEGHEM est maître de conférences à l'Université de Besançon où il enseigne la gestion de production et le marketing. Ses travaux de recherche conduits au sein de l'Équipe de recherche sur la firme et l'industrie (ERFI) portent sur l'assurance qualité en PME et sur le profil du dirigeant de PME. Adresse : Faculté de droit et de sciences économiques, Université de Besançon, Avenue de l'Observatoire, 25030 Besançon Cedex, France. 


\section{RESUMEN}

El desarrollo de las normas ISO 9000 en el mundo afecta a todos los sectores de actividad. Los dirigentes de pequeñas empresas pueden cuestionar la pertinencia de estos modelos concebidos al principio para las relaciones de tipo librador/ subcontratista. Este artículo tiene por objetivo valorizar el impacto que la implantación de un modelo de aseguramiento de la calidad tiene en el sistema de gestión de la pequeña empresa. Demostraremos como la implantación del aseguramiento de la calidad puede llevar a desnaturalizar la pequeña empresa es decir poner en tela de juicio sus especificidades fundamentales.

\section{ZUSAMMENFASSUNG}

Die weltweite Entwicklung der ISO 9000 Normen hat einen grossen Einfluss auf alle Tätigkeitssektoren. Die Führungskräfte von kleinen und mittleren Unternehmen (KMU) können die Richtigkeit eines solchen Modells in Frage stellen, welches ursprünglich basierend auf Verbindungen von Bestellern und Zulieferern aufgestellt wurde. Ziel dieses Beitrages ist es, die Wirkung eines Modells der Qualitätssicherung anhand der Führung einer KMU aufzuzeigen. Es wird veranschaulicht, in welchem Masse die Anwendung eines solchen Qualitätssystems eine KMU verändern und die Kernkompetenzen wieder herstellen kann.

\section{Introduction}

Les PME se voient de plus en plus obligées à mettre en place des modèles d'assurance de la qualité depuis la fin des années 1980 et l'émergence des normes ISO 9000. On peut se demander si ces modèles ne peuvent pas contribuer à modifier les principales caractéristiques du système de gestion de la PME. Dans la littérature sur la petite entreprise, de nombreux auteurs ont insisté sur les spécificités du système de gestion des PME (Welsh et White, 1981 ; Marchesnay, 1982 ; Marchesnay, 1991 ; Julien, 1994 ; Ghobadian et Gallear, 1996). Pour apprécier ces spécificités, on peut s'appuyer sur la définition de la PME sous forme de continuum proposée par Julien (1994). Elle met en évidence six caractéristiques pour mieux cerner le concept de PME : la petite taille, la centralisation de la gestion, une faible spécialisation, une stratégie intuitive ou peu formalisée, un système d'information interne peu complexe ou peu organisé, un système d'information externe peu complexe. Cette conception permet de tenir compte de l'hétérogénéité du monde des PME tout en soulignant l'existence de traits communs. Ces caractéristiques largement reconnues dans la littérature sur la PME sont au cœur de ce que l'on peut appeler le paradigme de la PME spécifique. Ce paradigme fait l'objet de discussions relatives à son cadre de validité (Torrès, 1997, 1998 ; Messeghem, 1998). En effet, dans certains contextes, les PME peuvent connaître des changements de nature indépendamment de la taille. Ces contextes que l'on peut qualifier de dénaturant peuvent prendre la forme d'un recours au capital de risque (Stéphany, 1998), de la mise en place d'un système EDI/JÀT 
(Curvalle et Torrès, 1998) ou encore de la mise sous assurance qualité. La dénaturation peut être comparée aux changements que connaissent les PME en phases de croissance, largement décrits dans les modèles de métamorphose (D'Amboise et Muldowney, 1988 ; Godener, 1996) et dans les travaux de l'école d'Aston (Pugh et al., 1968, 1969).

Pour éviter que les critiques adressées au modèle de cycle de développement, concernant notamment son caractère déterministe, trouvent un écho dans le courant de la dénaturation, il faut admettre que la dénaturation n'est pas systématique et que le dirigeant joue un rôle actif dans ce processus. Ainsi, certaines entreprises, dans un contexte dénaturant, peuvent continuer à fonctionner comme des petites entreprises «spécifiques », dans la mesure où le dirigeant souhaite conserver un fonctionnement traditionnel marqué par une forte personnalisation des relations. Guilhon et Weill (1996) dans une enquête auprès de 15 entreprises engagées dans une démarche qualité (certification d'entreprise ou qualité totale) semblent indiquer que la dénaturation n'est pas systématique. Ces auteurs font apparaître deux situations d'entreprises (site 1 et site 2):

- un premier groupe composé d'entreprises qui mettent en œuvre des normes du type ISO 9002 afin d'améliorer le fonctionnement interne et réduire les coûts de non-qualité. «La démarche qualité, peu intégrée dans les processus organisationnels et stratégiques ne semble pas bouleverser l'organisation. L'objectif est de reproduire le système organisationnel en valorisant les techniques et les compétences existantes; d'ailleurs les dirigeants de ces PME estiment que le niveau de compétence n'a pas changé. » Les démarches qualité mises en œuvre par ces entreprises n'ont pas pour effet de remettre en cause la spécificité de ces PME;

- les PME du deuxième groupe sont des entreprises de taille moyenne qui se sont lancées dans une démarche qualité globale (voire totale). «L'objectif recherché dans la démarche actuelle vise à améliorer les performances et surtout à créer un avantage concurrentiel centré sur le développement et l'implication interne ainsi que sur l'expansion. » Il s'agit d'entreprises qui connaissent une véritable métamorphose pour reprendre l'image de Starbuck (1965). Les changements organisationnels observés dans ces entreprises sont de nature à remettre en cause profondément les spécificités de la petite entreprise. Dans ce cas précis, la thèse de la dénaturation semble se justifier. Néanmoins, comme le montrent ces auteurs, les entreprises qui appartiennent à ce groupe s'inscrivent dans une logique d'expansion ; dès lors, il devient particulièrement difficile $\mathrm{d}$ 'isoler les facteurs dénaturants.

Nous allons, dans le cadre de cette contribution, nous intéresser aux répercussions de l'adoption d'un modèle d'assurance de la qualité sur les spécificités du système de gestion de la PME et en particulier sur la structure organisationnelle. 
La mise en place de cette méthode peut s'accompagner d'un processus de bureaucratisation. Dubost (1994) évoque ce processus en PME à travers l'exemple du contrôle externe. Cependant, pour comprendre l'adoption d'un modèle d'assurance de la qualité, il ne faut pas se focaliser uniquement sur l'évolution de l'organisation, mais il faut également tenir compte du contexte dans lequel s'inscrit cette démarche, c'est-à-dire prendre en considération les autres dimensions du système de gestion (Marchesnay, 1991). Si l'environnement est susceptible d'expliquer le recours à un modèle d'assurance de la qualité, les relations de la PME avec son environnement peuvent également être affectées par l'engagement dans un processus de certification. De même, l'entrepreneur, à travers ses motivations, sa stratégie, peut également influencer le processus de certification.

Nous allons donc privilégier le triptyque entrepreneur-environnementorganisation, pour comprendre le processus d'adoption d'un modèle d'assurance de la qualité, comme l'indique le schéma suivant. Les doubles flèches montrent qu'il existe des interactions entre ces différentes composantes.

SCHÉMA 1

Le système de gestion de la petite entreprise et l'assurance qualité

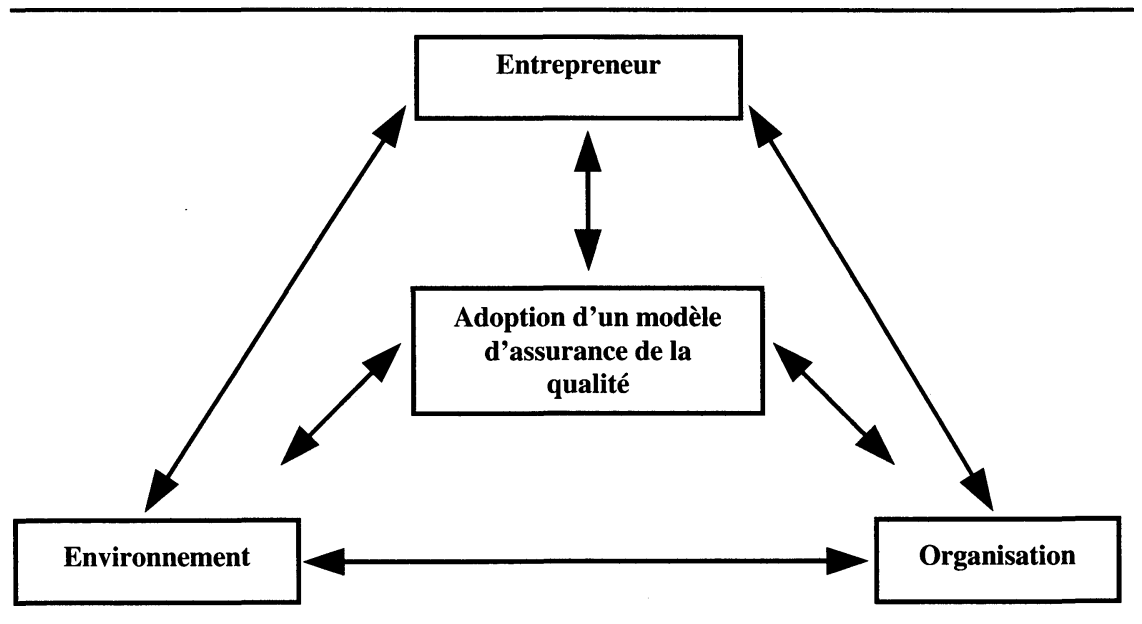

Nous nous intéresserons tout d'abord au profil du dirigeant en montrant que le succès d'une telle démarche repose sur sa capacité à faire partager sa vision à l'ensemble des partenaires de l'entreprise, mais également sur sa capacité à mobiliser suffisamment de ressources pour piloter ce nouveau projet. Nous verrons ensuite comment les évolutions de l'environnement peuvent inciter les PME à adopter un modèle d'assurance de la qualité, et comment cette méthode peut modifier les relations avec l'environnement. Nous montrons enfin que la mise en œuvre de cette démarche peut s'accompagner d'une bureaucratisation de la structure. 


\section{L'entrepreneur au cœur de la démarche}

Dans la littérature sur la PME, le dirigeant est très souvent placé au cœur des processus de changement organisationnel (Guilhon, 1993 ; Raymond et Blili, 1994 ; Sammut, 1995). Le dirigeant peut donc apparaître comme un personnage clé dans le processus de certification. Il doit être capable de construire un projet cohérent qui «s'évalue en fonction du lien établi entre la logique d'action du propriétaire dirigeant et les activités visées » (Marchesnay, 1997). Cette cohérence est largement influencée par le profil du dirigeant comme le suggère Marchesnay (1997). En effet, le projet de certification s'inscrit dans sa vision stratégique qu'il doit être capable d'expliciter pour essayer de la faire partager par l'ensemble des membres de l'organisation (Messeghem et Varraut, 1998). Le passage de la vision stratégique à l'intention stratégique suppose la combinaison de moyens et de buts (Varraut, 1998). Le dirigeant doit donc réussir à mobiliser des ressources pour mettre en œuvre son projet.

\subsection{La décision d'adoption d'un modèle d'assurance de la qualité}

En s'appuyant sur la réflexion de Julien et Marchesnay (1988) sur les aspirations des dirigeants, on peut distinguer deux principales logiques d'adoption des modèles d'assurance de la qualité : une logique patrimoniale et une logique managériale.

La logique patrimoniale : le dirigeant souhaite conserver le contrôle de son entreprise à long terme. Il se place dans une logique d'accumulation du capital. La structure du capital est caractéristique, elle est marquée par la faiblesse, voire l'absence de dettes financières à long terme. Cette logique de valorisation du capital va conduire le dirigeant à privilégier les investissements matériels et à éviter les investissements immatériels. On peut penser que l'assurance qualité ne sera adoptée par ce type de dirigeant que lorsque ses principaux concurrents se seront eux-mêmes lancés dans la démarche ou lorsque les exigences de ses clients sur cette question seront explicites, de façon à maintenir la survie de son entreprise. L'assurance qualité s'inscrira donc dans une stratégie plutôt adaptative. Sa recherche d'indépendance peut le conduire à limiter les effets de la mise sous assurance qualité que se soit en matière de centralisation, de formalisation ou de spécialisation. On peut faire l'hypothèse que ce type de dirigeant va essayer de conserver les spécificités liées à la taille de son entreprise. La mini-étude de cas suivante éclaire cette logique patrimoniale qui favorise les stratégies réactives.

La logique managériale : l'entrepreneur recherche avant tout des activités à forte croissance afin d'obtenir une forte rentabilité à court terme. Il privilégie une ouverture du capital et n'hésite pas à recourir à l'endettement pour bénéficier d'un effet de levier positif. Toutefois, il tient à conserver l'autonomie de décision. Ce type d'entrepreneur va s'intéresser à la certification d'entreprise dans la mesure où elle est susceptible de favoriser la croissance. La certification d'entreprise s'inscrira 


\section{Mini-étude de cas : La société Chabernac}

M. Chabernac a grandi dans les ateliers de l'entreprise familiale située en plein centre de Montpellier. Dans les années 1950, après avoir décroché son baccalauréat, il rejoint son père. L'entreprise spécialisée dans la fabrication de dragées et de gommes compte alors une dizaine de salariés. M. Chabernac prend la tête de l'entreprise, en 1965, à la suite du décès de son père. Il a toujours cherché à conserver cette image d'entreprise de tradition créée en 1880. Dans les années 1970, les locaux devenant vétustes, M. Chabernac décide de s'installer à la périphérie de Montpellier. Cet investissement permet d'accroître les capacités de production de l'entreprise qui comprend aujourd'hui une vingtaine de salariés. Le dirigeant s'est entouré de ses deux enfants. Son fils s'occupe de la production et sa fille des problèmes commerciaux. M. Chabernac continue à être très présent dans l'entreprise. Il a entamé une démarche d'assurance de la qualité dans le domaine de la production. Il a été incité à se lancer dans cette démarche par les audits répétés d'une grande entreprise de distribution alimentaire. $\mathbf{M}^{\mathrm{lle}}$ Chabernac souhaiterait que cette démarche soit étendue à l'ensemble de l'entreprise. M. Chabernac sait que son entreprise devra très certainement adopter un modèle d'assurance de la qualité dans les cinq ans, mais il préfère attendre que cette démarche se généralise dans son secteur d'activité. En effet, il redoute que cet investissement pénalise ses coûts de revient par rapport à ses principaux concurrents, et notamment par rapport aux produits provenant du sud de l'Europe. Il a le sentiment que cette méthode de gestion de la qualité est lourde à mettre en œuvre et difficile à rentabiliser.

davantage dans une stratégie de type proactif. En effet, le dirigeant n'attend pas que cette méthode s'impose ou que toutes les entreprises l'adoptent. Le fait d'obtenir un certificat d'entreprise apparaît pour lui comme un élément de différenciation. La mise aux normes peut également constituer un moyen pour exercer un meilleur contrôle sur son organisation. Cette stratégie peut notamment s'observer lors d'une succession ou d'un rachat. En effet, cette forme de gestion de la qualité peut contribuer à formaliser les savoir-faire au sein de l'organisation, mais elle peut surtout permettre de préciser les responsabilités de chacun. La dénaturation risque alors d'être plus marquée. Le dirigeant peut en effet essayer de développer à côté du système qualité un système de planification et de contrôle très formalisé étendu à l'ensemble des processus de l'entreprise. La mini-étude de cas La Conserverie Catalane permet d'illustrer la logique managériale d'adoption d'un modèle d'assurance de la qualité.

Derrière ces logiques d'adoption d'un modèle d'assurance se cache un processus de décision complexe caractérisé par l'articulation entre vision stratégique, intention stratégique et comportement (Messeghem et Varraut, 1998). Le projet de certification occupe une place plus ou moins importante dans la vision stratégique du dirigeant. On peut définir la vision comme une représentation de l'entreprise 


\section{Mini-étude de cas : La Conserverie Catalane}

M. Thevenet a racheté en 1991 la société La Conserverie Catalane, entreprise familiale d'une vingtaine de salariés. Cet ancien conseil en gestion d'entreprise n'a pas hésité à ouvrir le capital de son entreprise à un groupe financier présent dans l'agroalimentaire, notamment pour financer son développement. La Conserverie Catalane est une entreprise spécialisée dans la conserve de fruits. Elle a évolué, après ce rachat, vers la fabrication de conserves biologiques. Cette entreprise était caractérisée par un excellent savoir-faire, mais également par une forte culture orale. M. Thevenet a décidé de se lancer dans une démarche d'assurance de la qualité pour essayer de formaliser l'ensemble de ces savoir-faire. Il craignait que des départs de salariés puissent se traduire par une perte de savoir-faire. Il a rencontré une forte hostilité lors du lancement de cette démarche. Il a conduit une politique de formation destinée à sensibiliser l'ensemble des membres de l'organisation. Sa tâche a particulièrement été difficile au cours des deux premières années. En effet, durant cette période, le travail des salariés est alourdi sans qu'il y ait de retour sur investissement, ce qui peut être source de démotivation. Ce dirigeant a également souhaité mettre en place un système d'assurance de la qualité pour tenir compte des attentes de ses clients. Ses produits sont présents dans les épiceries fines mais également en grande distribution sous marque de distributeurs. L'exportation représente $20 \%$ de son activité (part qui devrait doubler dans les trois ans), essentiellement dans les pays du nord de l'Europe où les produits biologiques connaissent un grand succès. $M$. Thevenet a le sentiment que son entreprise dispose d'un avantage concurrentiel. En effet, le nombre d'entreprises certifiées ISO 9002 dans ce secteur est très faible. Mais selon lui, cet avantage repose également sur la qualité de ses produits qui sont des produits du terroir élaborés grâce à un savoirfaire reconnu et disposant d'une certification produit (Agriculture biologique).

dans le futur. Elle peut présenter une dimension onirique importante comme le suggèrent Hamel et Prahalad (1989). Selon ces deux auteurs, la vision doit faire naître des ambitions disproportionnées par rapport aux ressources et compétences détenues par l'entreprise. Cet écart est source de tension créatrice. Ainsi, selon Meschi (1997), « plus cet écart est grand, plus l'entreprise est amenée à développer et à combiner ses compétences de manière audacieuse pour le combler ».

Cet état de tension évoqué par Bird (1988) dans le cadre des décisions entrepreneuriales dépend de l'horizon de la décision, et plus exactement du temps disponible pour prendre la décision. Cette tension ne doit pas être trop importante, car elle peut être source d'anxiété et d'inhibition (Messeghem et Varraut, 1998). Si le décalage entre la représentation du présent et la représentation du futur est trop grand, la mise en œuvre de cette démarche risque de se heurter à deux écueils :

- d'une part, l'intention stratégique du dirigeant peut être irréaliste, ce qui va se traduire par un manque d'implication de la direction. Or, le succès 
de cette démarche repose largement sur l'engagement du dirigeant. Les modèles d'assurance de la qualité comprennent un chapitre consacré à la responsabilité de la direction (4.1). Ce chapitre prévoit que la direction doit élucider sa vision en mettant notamment par écrit sa politique de qualité.

- d'autre part, le personnel risque de témoigner une certaine indifférence, voire un rejet pour ce projet. Le passage entre vision stratégique et intention est donc conditionné par la faisabilité perçue du projet. Ainsi, un dirigeant qui ne dispose pas d'un système qualité très élaboré peut très bien percevoir l'assurance qualité comme une opportunité, mais être dissuadé de se lancer dans cette démarche par crainte de s'imposer un système lourd et coûteux.

Après avoir élucidé sa vision, le dirigeant doit donc essayer de susciter l'adhésion des membres de l'organisation. Selon la littérature sur la gestion de la qualité (Quiqley, 1996 ; Makita, 1992 ; Deming, 1996), lorsque le dirigeant essaie de mettre en œuvre une méthode de gestion de la qualité, il doit être en mesure d'obtenir l'assentiment de l'ensemble des membres de l'organisation. Sa tâche est rendue particulièrement ardue lorsque la vision est ambitieuse, mais surtout lorsqu'elle s'oppose à la culture de l'entreprise. L'adoption d'un modèle d'assurance de la qualité en petite entreprise peut s'inscrire dans ce contexte. En effet, pour une petite entreprise, la mise en place d'un système d'assurance de la qualité peut marquer le passage d'une logique processuelle à une logique procédurale (Julien et Marchesnay, 1992). La rédaction des procédures peut être perçue par les salariés comme un risque de «rigidification » et une perte de flexibilité. L'adoption d'un modèle d'assurance de la qualité peut également être perçue comme une remise en cause des rôles de chacun au sein de l'organisation et, par conséquent, se heurter à des enjeux de pouvoir (Mispelblom, 1995). Toutefois, la proximité du dirigeant avec les autres membres de l'entreprise peut simplifier sa tâche. Schein (1990) insiste sur le rôle du leader dans l'élaboration et dans le changement de culture, particulièrement dans les entreprises de petite taille.

\subsection{Des ressources difficiles à mobiliser}

Pour une petite entreprise, la mise en place du projet d'assurance de la qualité constitue un véritable investissement immatériel (Guilhon, 1993). Cette démarche conduit à mobiliser un certain nombre de ressources, et plus particulièrement des ressources financières et du temps.

\subsubsection{Les ressources financières}

La faiblesse des ressources financières est très souvent présentée comme l'une des caractéristiques de la petite entreprise (Beaudoin et St-Pierre, 1994). La mise sous assurance qualité présente un risque financier lié à son coût et à son irréversibilité. 
Le coût selon les évaluations varie entre $500000 \mathrm{~F}$ et $2000000 \mathrm{~F}$ (Couret et al., 1995 ; Berny et Peyrat, 1995). La partie visible comprend le recours à des cabinets conseils, à un organisme de certification, l'embauche d'un responsable qualité. Les « coûts cachés » sont les coûts liés à l'implication du dirigeant et des membres de l'organisation dans la définition du système qualité. La définition des procédures nécessite la mise en place de groupes de projet plus ou moins formalisés. Le temps consacré à la définition du système d'assurance qualité et à sa mise en place représente entre 18 et 24 mois. Pendant cette période, l'ensemble des membres de l'organisation est mis à contribution. Cette phase est comparable à la phase de démarrage (Sammut, 1995) dans la mesure où l'entreprise doit faire évoluer son système de gestion tout en devant faire face à ses activités quotidiennes. Dans le domaine de la production, l'instauration de nouvelles procédures peut réduire momentanément la productivité. En effet, elles peuvent allonger les temps de fabrication à travers, par exemple, l'introduction de nouvelles procédures de contrôle. La relation entre qualité (au sens de l'assurance de la qualité) et productivité est donc complexe, comme le souligne Treillon (1995).

Les dirigeants de petite entreprise ont très souvent du mal à mobiliser des ressources financières d'origine externe. Cela peut traduire la volonté du dirigeant qui souhaite conserver son indépendance (Julien et Marchesnay, 1988), mais cela traduit également un manque de crédibilité. Sammut (1995) évoque ce manque de crédibilité lors de la phase de démarrage. En effet, très souvent les dirigeants ont tendance à faire des prévisions optimistes, à minimiser l'importance des délais de paiement accordés, alors qu'eux-mêmes obtiennent difficilement des délais de paiement de leurs fournisseurs. La problématique n'est plus la même lorsque l'entreprise se lance dans une démarche de certification d'entreprise. En effet, elle s'engage à mettre en œuvre un système de gestion plus rigoureux afin d'améliorer la confiance à l'égard de ses clients et de ses partenaires. Certaines banques proposent des produits adaptés ; par exemple, le Crédit Lyonnais a lancé une expérience de financement de la démarche qualité des petites et moyennes entreprises à travers une enveloppe de financement baptisée « Action qualité 9000 » (Fouquet, 1994). Ce financement s'articule avec les aides mises à la disposition des PME. Ces entreprises peuvent bénéficier de trois types d'aides : le financement du diagnostic qualité ( $80 \%$ des frais d'intervention du consultant plafonnés à $25000 \mathrm{~F}$ ), le financement de la mise à niveau $(50 \%$ des frais engagés, plafonnés à $70000 \mathrm{~F}$ ou $100000 \mathrm{~F}$ ) et la prise en charge d'un poste de responsable qualité ( $50 \%$ du salaire et des charges sociales d'un cadre pendant un an, plafonnés à $150000 \mathrm{~F}$ ).

Le dirigeant peut essayer de diminuer le coût de la certification en s'inscrivant dans une démarche collective. Ainsi, dans la région Languedoc-Roussillon, de grands donneurs d'ordres du secteur de l'électronique tels que EDF et IBM ont encouragé des PME à se lancer dans l'assurance qualité. Une démarche collective 
a été conduite dans le cadre du Qualipôle, qui s'est notamment traduite par la mise en place d'actions de formation. Une dizaine d'entreprises ont ainsi décroché une certification d'entreprise en réduisant leur investissement.

\subsubsection{Le temps}

Les ressources financières constituent une dimension importante du système de gestion lors de phases critiques telles que la création ou la certification d'entreprise. Toutefois, à côté des ressources financières, il faut également tenir compte du temps, car en PME « la ressource la plus rare, susceptible de jouer le rôle de pivot sera le temps », comme le soutient Marchesnay (1995). Or le processus de certification s'inscrit dans une durée assez longue (entre 18 et 24 mois). Ainsi, même si l'entreprise dispose de moyens suffisants pour nommer un responsable qualité, il n'en demeure pas moins que tous les membres de l'organisation seront amenés à participer à cette démarche. Le dirigeant de petite entreprise va jouer un rôle important notamment lors de la phase de lancement. Il est le principal promoteur du changement de culture. Cette tâche ardue peut difficilement être déléguée au responsable qualité. Le dirigeant doit en effet justifier la démarche auprès de son personnel et expliquer pourquoi l'entreprise va recourir davantage aux procédures écrites. Cette tâche va donc occuper une partie de son emploi du temps. Le dirigeant sera ainsi amené à assurer de façon simultanée la direction de son entreprise et la mutation organisationnelle que peut représenter la certification.

Certains auteurs (Brune, 1993 ; Cruchant, 1995) semblent pourtant être d'avis que la mise en place d'un système d'assurance qualité pose moins de difficultés dans une petite entreprise, en raison de la simplicité de son système de gestion. Ces auteurs ont une représentation de la petite entreprise réductrice. Les tenants de la spécificité insistent au contraire sur l'effet loupe qui rend particulièrement difficile l'adoption de nouvelles méthodes de gestion. Bayad et al. (1995) parlent de «phénomène de grossissement des actions en PME ». Cet effet de grossissement (Mahé de Boislandelle, 1996) tend à amplifier les conséquences des problèmes qui se posent. Ainsi, la mise en place d'une démarche qualité présente un risque organisationnel lié à l'insatisfaction de certains employés. Cette insatisfaction peut se traduire par un allongement du processus de certification et peut même donner lieu dans certains cas à des départs de personnes qui ne se reconnaissent plus dans cette nouvelle organisation.

Les difficultés rencontrées peuvent freiner le processus de certification. Or, l'enlisement dans la démarche peut être négatif pour l'image de l'entreprise face à l'ensemble de ses partenaires, notamment par rapport à ses partenaires financiers et à ses clients. L'entreprise peut toutefois décider de ne pas aller jusqu'à la certification d'entreprise et se contenter de mettre en place un système d'assurance de la qualité proche des exigences des normes ISO 9000. Le dirigeant a alors tout intérêt à préciser 
son intention à l'ensemble des parties prenantes pour éviter une perte de motivation et de crédibilité. Ce risque a été traité dans les travaux consacrés aux cercles de qualité (Tang, Tollison et Whitesside, 1987 ; Hill, 1991 ; Makita, 1996).

\section{L'environnement}

L'adoption de modèles d'assurance de la qualité peut s'expliquer par la nature de l'environnement. Ainsi, dans les industries de sous-traitance, la mise en place de modèles d'assurance de la qualité en PME traduit essentiellement une réponse à des exigences formulées par de grands donneurs d'ordres (Froman, 1986 ; Gava, 1996 ; Gorgueu et Mathieu, 1996 ; Cogordan et Weill, 1998). L'assurance qualité s'inscrit alors dans le prolongement du contrôle qualité (Baudry, 1995). Cependant, dans d'autres secteurs d'activité, l'assurance qualité peut tout à fait s'inscrire dans une stratégie proactive (Messeghem et Varraut, 1998). L'entreprise peut alors chercher à instaurer une relation de confiance avec ses clients qui peut prendre la forme d'un partenariat. Cela suppose que l'entreprise dispose d'une bonne connaissance des attentes de ses clients. Pour mieux cerner leurs attentes et leur degré de satisfaction, l'entreprise peut être amenée à développer un système d'information externe, à côté du système d'information interne.

\subsection{La nature de l'environnement}

Si nous rejetons la thèse d'un déterminisme environnemental, en considérant que cette démarche peut s'inscrire dans une stratégie proactive, nous n'écartons toutefois pas l'idée d'une relation entre l'environnement et l'adoption d'un modèle d'assurance de la qualité. Pour évoquer cette relation, nous allons distinguer l'environnement sociétal et l'environnement concurrentiel.

La mise en place d'un système d'assurance de la qualité peut être influencée par l'environnement institutionnel, car elle peut constituer un moyen d'accroître la légitimité de l'entreprise. Les différents partenaires de l'entreprise en matière de qualité (organisations professionnelles, pouvoirs publics, organismes de certification) peuvent encourager les démarches de gestion de la qualité en général et la certification d'entreprise en particulier. De nombreuses régions sont à l'origine d'actions destinées à mieux faire connaître les méthodes de gestion de la qualité. Le fait d'appartenir à un réseau de pairs peut également favoriser l'adoption d'un modèle d'assurance de la qualité. En effet, cela peut constituer un moyen d'accéder à certaines informations, de bénéficier d'expériences (Filion, 1991). Des travaux (Young et Welsch, 1983 ; Gibb, 1997) ont d'ailleurs montré que les dirigeants de PME ont tendance à privilégier ce mode de collecte de l'information.

L'évolution de la réglementation, notamment sur le plan européen, conduit à une plus grande responsabilisation des industriels. Cette responsabilité pénale et 
civile se traduit par une diminution de la responsabilité des organismes de répression. Les entreprises doivent donc apporter la preuve en cas d'accident que tout a été mis en œuvre pour assurer la qualité. Ainsi, la directive européenne 93 / 43 CEE,

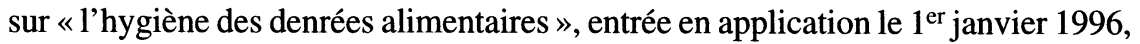
oblige les entreprises agroalimentaires à mettre en œuvre des autocontrôles selon les principes de la méthode $\mathrm{HACCP}^{1}$ (Analyse des risques et des points critiques pour la maîtrise). Pour exercer cette responsabilité, les fabricants doivent utiliser des outils spécifiques de maîtrise de la sécurité alimentaire. Cette directive propose notamment de recourir à l'assurance qualité sous sa forme certification d'entreprise. Cette démarche garde un caractère volontaire. Cependant, le fait qu'un texte réglementaire encourage la mise sous assurance qualité réduit la marge de manœuvre des dirigeants de petites entreprises.

Si l'on raisonne par rapport à l'environnement concurrentiel, l'assurance qualité est susceptible de modifier le risque de dépendance et de vulnérabilité. Le fait d'adopter un modèle d'assurance peut constituer un avantage concurrentiel lorsque peu de firmes comparables se sont lancées dans la démarche et surtout lorsque le client est sensible aux démarches qualité adoptées par ses fournisseurs. La certification peut également constituer, pour des petites entreprises locales, un moyen d'accéder à de nouveaux débouchés au plan national mais surtout au plan international (Gunasekaran et al., 1996). Ainsi, dans les industries agroalimentaires, les démarches de certification d'entreprise constituent une clé d'entrée indispensable sur les marchés anglo-saxons, à l'origine de la certification d'entreprises, mais aussi dans la grande distribution. L'adoption d'un modèle d'assurance de la qualité peut constituer une étape dans l'établissement de relation de partenariat. Cependant, si la concurrence se place uniquement sur les prix, l'entreprise certifiée va supporter un surcoût qu'elle ne pourra pas répercuter sur le prix de vente. L'assurance qualité peut également apparaître comme un investissement immatériel spécifique. En effet, dans certaines industries telles que l'automobile, les grands donneurs d'ordres continuent à imposer des exigences spécifiques en matière de contrôle, d'échange des données. Ces exigences spécifiques sont reprises dans les modèles d'assurance de la qualité qu'imposent les grands constructeurs. L'assurance qualité est donc susceptible d'accroître la vulnérabilité et la dépendance des petites entreprises à l'égard de leurs clients. Dans cette conception, l'assurance qualité n'apparaît que comme un modèle de sélection des fournisseurs (Messeghem, 1999).

26. HACCP (Hazard Analysis Critical Control Point) : méthode née aux États-Unis à la fin des années 1960 dans le cadre du programme aérospatial et destinée à assurer la sécurité alimentaire des cosmonautes. Cette méthode s'intéresse aux processus et au produit à travers la mise en évidence des risques biologiques, chimiques et physiques, et l'élaboration d'un plan de contrôle. 


\subsection{Vers une formalisation du système d'information externe}

Les entreprises doivent être à l'écoute de l'environnement pour assurer notamment la cohérence entre le métier et la mission (Marchesnay, 1993). Cela suppose que l'entreprise dispose d'un système d'information externe plus ou moins formalisé. En petite entreprise, la personnalisation des relations permet le plus souvent de conserver un système d'information externe peu formalisé. Les trois modèles d'assurance qualité n'imposent pas la mise en place d'un système d'information externe formalisé. En effet, aucun de ces modèles ne fixe d'exigences relatives à la collecte et à la gestion d'information d'origine externe. Les relations avec l'environnement tendent à être éludées. Ces modèles font essentiellement référence aux attentes des clients qui apparaissent comme une donnée exogène. Elles sont reprises au sein de l'organisation sous forme de spécifications. Cette position s'explique par les origines de la certification d'entreprise qui trouve ses racines dans les relations de sous-traitances où très souvent les spécifications étaient imposées aux sous-traitants sous forme de cahier des charges.

Cependant, lorsqu' on étudie les lignes directrices et plus particulièrement la norme ISO 9004-1, on peut découvrir des paragraphes consacrés au système d'information externe. Ainsi, la norme ISO 9004-1 relative au « Management de la qualité et éléments de système» consacre un chapitre à la "qualité en mercatique». Rappelons que la norme ISO 9004 ne fixe pas d'exigences et qu'elle «n'est pas destinée à une utilisation contractuelle, réglementaire ou de certification ». Les entreprises qui souhaitent s'inspirer de la directive sur la qualité en mercatique sont conduites à mettre en œuvre un système d'information relativement élaboré, voire complexe. En effet, cette directive précise qu' " il convient que la fonction mercatique définisse et consigne par écrit les exigences qui s'imposent pour la qualité du produit ». La mise en place des normes n'implique donc pas systématiquement l'élaboration d'un système d'information externe complexe. Il semble donc pertinent de se demander si l'une des particularités des petites entreprises certifiées n'est pas de conserver un système d'information externe peu complexe et fortement centralisé.

\section{Une « rigidification » de l'organisation}

Si les modèles d'assurance de la qualité ne définissent pas d'exigences relatives au système d'information externe, il en va autrement pour les systèmes d'information interne. En effet, ces normes imposent la mise en place d'un système documentaire destiné à consigner les procédures et à assurer le suivi des actions dans le domaine de la qualité. Le système d'assurance peut également modifier la structure de l'entreprise, en favorisant le passage d'une structure simple à une structure bureaucratique (Mispelblom, 1995). 


\subsection{Complexification du système d'information interne}

L'assurance de la qualité conduit à mettre en place un système d'information interne formalisé. Ce système d'information risque très vite de devenir compliqué et très organisé. Les normes ISO 9000-2 définissent certaines activités destinées à assurer la maîtrise des données relatives à la qualité au sein de l'organisation. L'accent est mis sur les savoirs formalisés. La norme semble suggérer qu'il est possible de formaliser l'ensemble des savoirs. Cette conception de la norme trouve un terrain de prédilection dans des formes d'organisation de type mécanique, bureaucratique comme l'exprime Reix (1996) : «Considérer que la connaissance explicite est préférable, c'est construire une organisation où la formalisation puis la combinaison des connaissances formelles sont privilégiées, où l'essentiel de la connaissance est exprimé selon un code partagé et donc susceptible d'être séparé des individus qui la détiennent. Cela conduit à des formes d'organisation de type mécanique, bureaucratique.»

L'objectif des modèles d'assurance est de formaliser «la mémoire de l'entreprise » relative à la maîtrise de la qualité. Cette volonté de formaliser tous les savoirs et savoir-faire qui ont une incidence sur la qualité est exprimée dans l'ensemble des points de la norme. Le chapitre (4.5) de la norme consacré à la «maîtrise des documents et des données » prévoit que l'entreprise doit définir et formaliser des procédures relatives à la gestion du système d'information: «Avant leur diffusion, les documents et les données doivent être revus et approuvés en ce qui concerne leur adéquation, par des personnes habilitées. Une liste de référence ou toute procédure de maitrise de documents équivalents indiquant la révision en vigueur des documents doit être établie et être facilement accessible pour empêcher l'utilisation de documents non valables ou périmés. »

Ce chapitre précise quelles sont les conditions d'accès et les conditions de modifications des différents documents disponibles dans l'entreprise. Le chapitre 4.16 consacré à la «maîtrise des enregistrements relatifs à la qualité » oblige les entreprises à conserver l'ensemble des données permettant d'apporter la preuve que l'entreprise a respecté les exigences relatives à la norme. Cette maîtrise des enregistrements est destinée à apporter des éléments de preuve, lorsque le client subit un préjudice après avoir utilisé un produit défectueux. La norme privilégie le développement d'un système d'information qualité centré sur le produit. Ainsi, le paragraphe 4.8 «Identification et traçabilité du produit» incite les entreprises à assurer un suivi du produit. Dans certains secteurs tels que l' agroalimentaire l'industrie pharmaceutique, la traçabilité constitue une exigence.

La place du dirigeant au cœur du système de gestion de l'entreprise peut être un facteur de succès dans la mise en place d'un système d'information qualité. Le dirigeant peut également contribuer à atténuer la complexification du système d'information interne. Raymond et Blili (1994) défendent cette idée : « La PME est 
caractérisée par une structure simple très centralisée. La centralisation peut être vue comme un facteur de succès dans l'implantation de systèmes d'information organisationnels, car elle réduit la complexité. »

\subsection{Une structuration des activités}

L'objectif de la certification est d'assurer une plus grande régularité par une meilleure maîtrise des processus. Selon Mintzberg (1982), « les organisations formalisent le comportement pour en réduire la variabilité, et en fin de compte pour le prédire et le contrôler ». On peut donc s'interroger sur l'évolution de la structure à la suite de l'adoption d'un modèle d'assurance de la qualité. On peut se demander si les petites entreprises qui adoptent un système d'assurance de la qualité «n'évoluent pas vers des bureaucraties ». Dubost (1994) pose cette question en s'intéressant au contrôle externe exercé sur les PME. Pour apprécier ce phénomène de bureaucratisation, on peut s'appuyer sur les travaux du groupe d'Aston (Pugh et al., 1968, 1969). Comme nous avons déjà eu l'occasion de le souligner, ces travaux montrent qu'il est possible de définir les bureaucraties à partir d'une variable : la structuration des activités. Pugh et al. (1968) proposent pour définir la structuration des activités de retenir trois principales dimensions : la spécialisation, la formalisation et la standardisation. On peut essayer de relier ces trois dimensions aux différents chapitres de la norme, comme l'illustre le tableau suivant.

\section{TABLEAU 1}

Norme ISO 9001 et structuration des activités

\begin{tabular}{|c|c|c|c|}
\hline Variables & Standardisation & Formalisation & Spécialisation \\
\hline Définition & $\begin{array}{l}\text { Existence de procédures } \\
\text { et de règles }\end{array}$ & $\begin{array}{l}\text { Recours à l'écrit (règles } \\
\text { et communication) }\end{array}$ & $\begin{array}{l}\text { Division du travail au } \\
\text { sein de l'organisation }\end{array}$ \\
\hline $\begin{array}{l}\text { Exigences de la } \\
\text { norme ISO } 9001\end{array}$ & $\begin{array}{l}\text { "Le fournisseur doit } \\
\text { établir et tenir à jour } \\
\text { des procédures écrites } \\
\text { pour maîtriser tous les } \\
\text { documents et données } \\
\text { relatives aux exigences } \\
\text { de la présente norme } \\
\text { internationale. " }\end{array}$ & $\begin{array}{l}\text { "Le fournisseur doit } \\
\text { établir un manuel qualité } \\
\text { couvrant les exigences } \\
\text { de la présente norme } \\
\text { internationale." }\end{array}$ & $\begin{array}{l}\text { "La responsabilité, } \\
\text { l'autorité et les relations } \\
\text { entre les personnes qui } \\
\text { dirigent, exécutent et } \\
\text { vérifient des tâches qui } \\
\text { ont une incidence sur la } \\
\text { qualité doivent être } \\
\text { définies par écrit." }\end{array}$ \\
\hline & $\S 4.5 .1)$ & $(\S 4.2 .1)$ & $(\S 4.1 .2 .1)$ \\
\hline
\end{tabular}

Ce tableau montre que l'adoption d'une norme du type ISO 9001 peut s'accompagner d'une bureaucratisation de l'organisation. Cette bureaucratisation touche-t-elle l'ensemble du système de gestion de l'entreprise ou est-elle limitée au seul système qualité ? Le chapitre de la norme consacré à la maîtrise des processus (4.9) implique la mise en place de procédures concernant l'ensemble des 
processus ayant une incidence sur l'obtention de la qualité : «Le fournisseur doit identifier et planifier les processus de production, d'installation et les processus relatifs aux prestations associées qui ont une incidence directe sur la qualité, et il doit aussi assurer que ces processus sont mis en cuvre dans des conditions mâेtrisées. » Il paraît toutefois difficile de circonscrire cet accroissement de standardisation au seul système qualité.

On peut également s'interroger sur l'évolution de la centralisation lors de l'adoption d'un modèle d'assurance de la qualité. La bureaucratisation ne s'accompagne pas nécessairement d'une décentralisation (Mansfield; 1973 ; Mintzberg, 1982). Le paragraphe 4.1 relatif à la responsabilité de la direction impose une définition claire des responsabilités en matière de qualité. Ce besoin de clarification peut se traduire par la nomination d'un responsable qualité et par la création d'un service qualité. Le responsable qualité dans la terminologie de Mintzberg (1982) est un analyste de la technostructure qui est susceptible de bénéficier d'une décentralisation sélective. Cette décentralisation risque d'être transitoire, c'est-à-dire liée au processus de certification. En effet, une fois que l'entreprise est certifiée, le travail du responsable qualité se limite à un suivi du système qualité et à l'exécution du contrôle qualité.

\section{Conclusion}

Nous avons souhaité dans cet article analyser les répercussions d'un processus de certification sur le système de gestion de la PME. Nous avons tout d'abord essayé de souligner l'importance du contexte dans lequel s'inscrit l'adoption d'un modèle d'assurance de la qualité. Nous avons retenu deux dimensions principales pour caractériser ce contexte : l'environnement et l'entrepreneur. Dans la littérature, la certification est très souvent présentée comme une contrainte imposée par les donneurs d'ordres. Nous avons au contraire insisté sur la dimension stratégique du projet certification en montrant que le dirigeant joue un rôle actif. En effet, ce projet peut s'intégrer dans sa vision stratégique, en étant perçu comme un moyen pour saisir de nouvelles opportunités.

Nous avons ensuite cherché à apprécier les répercussions de l'adoption d'un modèle d'assurance de la qualité sur le système de gestion de la PME, et en particulier sur la structure. Nous avons essayé de montrer que la mise sous assurance qualité peut constituer un facteur dénaturant. Il semble en effet que les changements que connaissent les PME engagées dans un processus de certification apparaissent comme une altération des spécificités de la PME. Cependant, si ces entreprises adoptent un mode de fonctionnement managérial, cela ne signifie pas qu'elles sont incapables de mettre en œuvre des stratégies entrepreneuriales. La capacité à combiner logique entrepreneuriale et logique managériale peut même constituer un facteur de performance pour ces PME. 
D'un point de vue épistémologique, le courant de la dénaturation ne vise pas à remettre en cause le paradigme de la spécificité, mais à en préciser le cadre de validité. En effet, tout paradigme est caractérisé par des limites au-delà desquelles l'homme de sciences est appelé à résoudre des énigmes (Chalmers, 1987). Le courant de la dénaturation se situe ainsi aux frontières du paradigme dans la mesure où il suscite de nouvelles énigmes non résolues qui constituent autant d'enjeux pour la recherche en PME.

\section{Bibliographie}

BAUDRY, B. (1995), L'économie des relations interentreprises, Paris, Éditions La Découverte.

Bayad, M., H. MahÉ de Boislandelle, D. Nebenhaus et P. Sarnin (1995), «Paradoxes et spécificités des problématiques de gestion des ressources humaines en petites et moyennes entreprises», Gestion 2000, $\mathrm{n}^{\circ} 1$.

Beaudoin, R. et J. ST-PierRe (1994), «Les problèmes financiers des PME : l'état de la question », dans P.A. Julien, Les PME : Bilan et perspectives, Paris, Economica.

BERNY, L. et O. PEYRAT (1995), «La certification d'entreprise : vrais enjeux et faux débats », Revue française de gestion, novembre-décembre.

BIRD, B.J. (1988), «Implementing entrepreneurial ideas : the case for intentions », Academy of Management Review, vol. 13, $\mathrm{n}^{\circ} 2$.

BRUNE, A. (1993), La certification : clé d'un nouvel essor économique, Rapport présenté aux ministres chargés de l'Industrie, de l'Agriculture et de la Consommation, février.

Chalmers, A.F. (1987), Qu'est-ce que la Science, Paris, Éditions La Découverte.

COGORDAN, J. C. et M. WEILL (1998), « Pour une stratégie de démarche qualité par la maîtrise

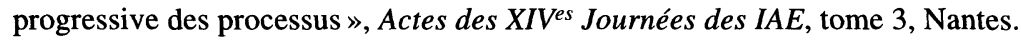

Couret, A., J. Igalens et H. Penan (1995), La certification, Paris, Presses universitaires de France.

Cruchant, L. (1995), La qualité, Paris, Presses universitaires de France, Collection « Que sais-je ?"

CurvalLE, B. et O. ToRRÈs (1998), «Le système EDI/JAT condamne-il les PME ? », dans $\mathrm{O}$. Torrès (dir.), PME : De nouvelles approches, Paris, Economica.

D'AMBoise, G. et M. MULDOWNEY (1988), « Management theory for small business : attempts and requirements », Academy of Management Journal, vol. 13, $\mathrm{n}^{\circ} 2$.

Deming, E. W. (1996), Du nouveau en économie, Traduction de l'ouvrage New Economics for Industry, Paris, Economica.

DuBost, N. (1994), «Les PME françaises évoluent-elles vers des bureaucraties ?», Communication au colloque de l'AIMS, Paris-Dauphine, décembre.

FILION, L.J. (1991), «Vision and relations : elements for an entrepreneurial metamodel », International Small Business Journal, vol. 9, $\mathrm{n}^{0} 2$.

FOUQUET, C. (1994), « Financer la démarche qualité : Un investissement de fonctionnement », Enjeux, $\mathrm{n}^{\circ} 148$, Éditions AFNOR, octobre. 
FROMAN, B. (1986), «L'harmonisation ISO des systèmes qualité » Enjeux, nº 72, Éditions AFNOR, septembre.

GAVA, M. J. (1996), «Les équipementiers dans la danse de la certification », Enjeux, n 169 , novembre.

GIBB, A.A. (1997), « Small firms' training and competitiveness. Building upon the small business as a learning organization », International Small Business Journal, vol. 15, $\mathrm{n}^{\circ} 3$.

GHOBADIAN, A. et D.N. GAlleAR (1996), « Total quality management in SMEs », Omega, vol. $24, \mathrm{n}^{\circ} 1$.

GODENER, A. (1996), La survenue des seuils organisationnels dans les petites et moyennes entreprises industrielles en croissance, Thèse en sciences de gestion, Université de Grenoble II.

GorgueU, A. et R. MATHIEU (1996), «L'assurance qualité fournisseur de l'industrie automobile française ", Revue d'économie industrielle, $\mathrm{n}^{\circ} 75$.

GuILHON, A. (1993), Étude de la relation entre le changement organisationnel et l'investissement intellectuel dans les PME, Thèse de doctorat en sciences de gestion, Université de Montpellier I.

Guilhon, A. et M. WeILl (1996), «Démarche qualité : De la stratégie d'adaptation aux processus stratégiques de changement dans les PME. Résultats d'un test empirique », Communication au colloque de l'AIMS, Lille.

Gunasekaran, A., P. OKKo, T. Martikainen et P. Yli-Olli (1996), «Improving productivity and quality in small and medium enterprises : cases and analysis ", International Small Business Journal, vol. 15, $\mathrm{n}^{\mathrm{0}} 1$.

HAMEL, G. et C.K. PRAHALAD (1989), «Strategic Intent », Harvard Business Review, mai-juin.

HILL, S. (1991), «Why quality circles failed but total quality management might succeed», British Journal of Industrial Relations, vol. 24, $\mathrm{n}^{\circ} 2$.

Julien, P.A. (1994), « Pour une définition des PME», dans P.A. Julien (dir.), Les PME: Bilan et perspectives, Paris, Economica.

JuliEn, P.A. et M. MARCheSNAy (1988), La petite entreprise, Paris, Vuibert.

JULIEN, P.A. et M. MARCHESNAY (1992), « Des procédures aux processus stratégiques dans les PME », Revue Picola Impresa, $\mathrm{n}^{\circ} 2$.

MAHÉ DE BoislANDELLE, H. (1996), «L'effet de grossissement chez le dirigeant de PME: incidences sur le management des ressources humaines », Gestion 2000, vol. 12, no 4.

MAKITA, P. (1992), Analyse des facteurs de performance des cercles de qualité en milieu industriel, Thèse de doctorat en sciences de gestion, Nanterre, Université de Paris X.

MAKITA, P. (1996), «La qualité en milieu industriel : Quel management? », Ve Conférence de l'AIMS, Lille.

MANSFIELD, R. (1973), «Bureaucracy and centralization : an examination of organizational structure », Administrative Science Quarterly, vol. 18.

MARCHESNAY, M. (1982), «Pour un modèle de l'hypofirme», dans Entreprise et organisation, Paris, Economica. 
MARCHESNAY, M. (1991), «La P.M.E. : Une gestion spécifique », Economie rurale, $\mathrm{n}^{\circ} 206$.

MARCHESNAY, M. (1993), Management stratégique, Paris, Eyrolles.

MARCHESNAY, M. (1995), «Information, risque et système de gestion de l'hypofirme », Communication au Deuxième Colloque international de la PME, Paris, octobre.

MARCheSNAY, M. (1997), «L'entrepreneuriat rural en quête de légitimités », Document de recherche, ERFI, juin.

MeSCHI, P.X. (1997), «Le concept de compétence en stratégie : Perspectives et limites », VI ${ }^{\mathrm{e}}$ Conférence de l'AIMS, Montréal, 1997.

MESSEGHEM, K. (1998), «Implications organisationnelles pour les PME de la mise sous assurance qualité », $4^{\mathrm{e}}$ Congrès international francophone sur la PME, Nancy, Metz.

MESSEGHEM, K. (1999), «Analyse des méthodes de gestion de la qualité dans les relations interentreprises », Gestion 2000 (à paraître).

MESSEGHEM, K. et N. VARRAut (1998), «Stratégies d'adoption d'une démarche qualité en $\mathrm{PME} »$, Revue internationale PME, vol. $11, \mathrm{n}^{\circ} 1$.

MinTZBERG, H. (1982), Structure et dynamique des organisations, Paris, Les Éditions d'Organisation.

MisPelblom, F. (1995), Au-delà de la qualité : Démarches qualité, condition de travail et politiques $d u$ bonheur, Paris, Syros, $288 \mathrm{p}$.

Pugh, D.S., D.J. Hickson, C.R. Hinings et C. TURNER (1968), «Dimensions of structure organization », Administrative Science Quarterly, vol. 13.

Pugh D.S., D.J. HiCKson, C.R. Hinings et C. Turner (1969), « The context of organization structures », Administrative Science Quarterly, vol. 14.

QuiQLeY, J.V. (1996), «Vision : how leaders develop it, share it, and sustain it», dans J.W. Cortada et J.A. Woods (dir.), The Quality Yearbook, New York, McGraw Hill, $782 \mathrm{p}$.

RAYMOND, L. et S. BLILI (1994), «Les systèmes d'information », dans P.A. Julien (dir.), Les PME : Bilan et perspectives, Paris, Economica.

REIX, R. (1996), «Savoir tacite et savoir formalisé dans l'entreprise », Revue française de gestion, septembre-octobre.

SAMMUT, S. (1995), Contribution à la compréhension du processus de démarrage en petite entreprises, Thèse de doctorat de sciences de gestion, Université de Montpellier I.

SCHEIN, E.H. (1990), «Organizational culture», American Psychologist, vol. 45, $\mathrm{n}^{\circ} 2$.

STARBUCK, W.H. (1965), « Organizational growth and development», dans J.C. March (dir.), Handbook of Organizations, Chicago, Rand McNally, 1247 p.

STÉPHANY, E. (1998), «Analyse de l'impact d'un financement par capital-risque sur l'organisation et le management de la $\mathrm{PME}$ », dans O. Torrès (dir.), PME : De nouvelles approches, Paris, Economica.

TANG, T.L.P., P.S. Tollison et H.D. WhiteSside (1987), « The effect of quality circle initiation on motivation to attend quality circle meetings and on task performance », Personnel Psychology, vol. 40, hiver. 
TORRÈS, O. (1997), «Pour une approche contingente de la spécificité de la PME », Revue internationale $P M E$, vol. $10, \mathrm{n}^{\circ} 2$.

TORRÈS, O. (dir.) (1998), PME: De nouvelles approches, Paris, Economica, 187 p.

TREILLON, R. (1995), «Qualité intégrale de l'entreprise et certification ou comment ne pas oublier la productivité », dans F. Nicolas et E. Valceschini (dir.), Agroalimentaire : une économie de la qualité, Paris, Economica.

VARRAut, N. (1998), «Pour une mise en relation des concepts de vision et d'intention stratégiques : Configurations de prises de décision stratégique », Actes des XIVes Journées des IAE, tome 3, Nantes.

WELSH, J. et J. WhITE (1981), «A small business is not a little big business », Harvard Business Review, juillet-août.

YOUNG, E.C. et H.P. WELSCH (1983), «Information source selection patterns as determined by small business problems », American Journal of Small Business, vol. 7, n 4 . 